\title{
Coordinated regulation of apical hook development by gibberellins and ethylene in etiolated Arabidopsis seedlings
}

\author{
Fengying $\mathrm{An}^{1}$, Xing Zhang ${ }^{1}$, Ziqiang Zhu ${ }^{1}$, Yusi $\mathrm{Ji}^{1}$, Wenrong $\mathrm{He}^{1}$, Zhiqiang $\mathrm{Jiang}^{1}$, Mingzhe $\mathrm{Li}^{1}$, Hongwei Guo ${ }^{1,2}$ \\ ${ }^{I}$ State Key Laboratory of Protein and Plant Gene Research, College of Life Sciences, Peking University, Beijing 100871, China; \\ ${ }^{2}$ Peking-Tsinghua Center for Life Sciences, Beijing 100871, China
}

Dark-grown Arabidopsis seedlings develop an apical hook when germinating in soil, which protects the cotyledons and apical meristematic tissues when protruding through the soil. Several hormones are reported to distinctly modulate this process. Previous studies have shown that ethylene and gibberellins (GAs) coordinately regulate the hook development, although the underlying molecular mechanism is largely unknown. Here we showed that GA $_{3}$ enhanced while paclobutrazol repressed ethylene- and EIN3-overexpression (EIN3ox)-induced hook curvature, and della mutant exhibited exaggerated hook curvature, which required an intact ethylene signaling pathway. Genetic study revealed that GA-enhanced hook development was dependent on HOOKLESS 1 (HLS1), a central regulator mediating the input of the multiple signaling pathways during apical hook development. We further found that $\mathbf{G A}_{3}$ induced (and DELLA proteins repressed) HLS1 expression in an ETHYLENE INSENSITIVE 3/EIN3-LIKE 1 (EIN3/ EIL1)-dependent manner, whereby EIN3/EIL1 activated $H L S 1$ transcription by directly binding to its promoter. Additionally, DELLA proteins were found to interact with the DNA-binding domains of EIN3/EIL1 and repress EIN3/EIL1-regulated HLS1 expression. Treatment with naphthylphthalamic acid, a polar auxin transport inhibitor, repressed the constitutively exaggerated hook curvature of EIN3ox line and della mutant, supporting that auxin functions downstream of the ethylene and GA pathways in hook development. Taken together, our results identify EIN3/EIL1 as a new class of DELLA-associated transcription factors and demonstrate that GA promotes apical hook formation in cooperation with ethylene partly by inducing the expression of HLS1 via derepression of EIN3/EIL1 functions.

Keywords: gibberellins; ethylene; EIN3/EIL1; HLS1; apical hook

Cell Research (2012) 22:915-927. doi:10.1038/cr.2012.29; published online 21 February 2012

\section{Introduction}

Sessile plants cannot move, so they can only alter their growth pattern through differential growth to adapt to the changing environment. In dicotyledonous plants, the first process of differential growth during their lifespan is the formation of an apical hook, which protects cotyledons and apical meristematic tissues from mechanical damage when elongating seedlings protrude through the soil to the surface [1]. The apical hook formation in Arabidopsis

Correspondence: Hongwei Guo ${ }^{\mathrm{a}}$; Fengying $\mathrm{An}^{\mathrm{b}}$

${ }^{a}$ E-mail: hongweig@pku.edu.cn

${ }^{b}$ E-mail: fyan1204@gmail.com

Received 19 May 2011; revised 7 October 2011; accepted 14 November 2011; published online 21 February 2012 is caused by differential cell growth on the opposite side of the hypocotyl, where the rates of cell elongation in the outside are faster than the inner side $[2,3]$. This process is coordinated by several hormones. Auxin has been extensively studied for its role in apical hook development. Exogenous application of auxin [4] or genetic manipulation of auxin-synthesis genes [5-7] causes defects in apical hook formation. Moreover, blocking polar auxin transport by treatment with naphthylphthalamic acid (NPA) prevents hook formation [4, 8]. An asymmetrical accumulation of auxin is thought to be necessary for the differential cell growth during hook development [8].

Besides the regulation by auxin, ethylene is another regulator of hook development. Exogenous treatment with ethylene or its biosynthesis precursor 1-aminocyclopropane-1-carboxylic acid (ACC) leads to the development of exaggerated apical hook, together with a short, 
thickened root and hypocotyl, which is known as the "triple response" of etiolated seedlings [9, 10]. A number of ethylene response mutants that show an aberrant triple response phenotype have been identified in Arabidopsis. Of these mutants, the etiolated seedlings of ethyleneinsensitive mutants, such as etr1 [11], ein2 [12], and ein3 [13], exhibit reduced apical hook, long hypocotyls and roots even in the presence of ethylene. In contrast, mutants resulting from either ethylene overproduction (etol) [12] or activated ethylene signaling (ctrl) [14] show constitutively exaggerated apical hook. Mutations in HOOKLESS 1 (HLS1), which encodes a protein with sequence similar to $N$-acetyltransferase, have also been identified that exhibit a complete loss of hook formation in the presence of exogenous ethylene application [8, $12]$. While the hls 1 mutant fully suppresses the phenotype of exaggerated hook curvature of ctrl or etol [15], overexpression of $H L S 1$ results in a constitutive hook curvature [8], indicating an essential role of HLS1 in the regulation of hook development.

Multiple hormone interactions have been revealed in the control of apical hook development, in which the cross-talk between auxin and ethylene was extensively studied. Ethylene was reported to activate the transcription of $H L S 1$, whose activity is required for the normal expression of auxin-responsive genes and the reporter gene DR5::GUS in the hook region $[3,8]$. Moreover, a genetic screen has identified AUXIN RESPONSE FACTOR 2 (ARF2), whose mutation suppresses the hls 1 phenotype. Ethylene downregulates the level of the ARF2 protein in a HLS1-dependent manner [3]. These studies pinpoint HLS1 as a key mediator of ethylene and auxin signaling in the regulation of the apical hook curvature. Meanwhile, two recent studies revealed direct regulation of the auxin polar transport machinery by ethylene through analyzing hook development kinetics $[16,17]$. They found that ethylene induces exaggerated hook curvature by activating the transcription of an auxin influx carrier AUX1, as well as by altering AUX1 protein distribution. Additionally, ethylene regulation of apical hook development also involves modulation of the activity of the PIN-dependent auxin efflux machinery through transcriptional and posttranscriptional mechanisms.

Another plant hormone gibberellins (GAs) were also reported to regulate the hook curvature. GA deficiency (gal-t) or treatment with paclobutrazol (PAC), an inhibitor of GA biosynthesis, inhibits ethylene-induced hook curvature of dark-grown seedlings [18], which is reversed by mutations resulting in defects in DELLAs [18]. DELLA proteins are key repressors of GA responses by inhibiting GA-regulated gene expression [19]. These repressors accumulate in the nucleus and are rapidly degraded via the 26S proteasome pathway in response to GAs $[20,21]$. Mutations within the DELLA domains make these proteins resistant to degradation and result in a GA-insensitive dwarf phenotype [22, 23]. It is thought that the DELLA repressors act negatively in GA responses by association with diverse transcription factors or regulators $[24,25]$, including the bHLH-family transcription factors PIF3 and PIF4 [26, 27], another bHLH protein ALCATRAZ (ALC) [28], and a GRAS family member SCL3 $[29,30]$. In addition, DELLA promotes jasmonic acid signaling by directly binding to and sequestering jasmonic acid-signaling repressors, JAZ proteins [31]. Accumulating evidence has demonstrated that DELLA proteins act as central regulators that control a wide array of plant growth and development processes. The identification of additional associated transcription factors would shed light on the distinct actions of DELLA proteins in various GA-regulated responses.

Here we show that ethylene and GAs coordinately enhance apical hook formation by inducing the expression of HLS1 in an ETHYLENE INSENSITIVE 3/EIN3LIKE 1 (EIN3/EIL1)-dependent manner. In addition, we find that HLS1 is a direct target gene of EIN3/EIL1, and that DELLA proteins inhibit the function of EIN3/EIL1 by associating with their DNA-binding domains. Therefore, our study provides new insight into the molecular mechanisms underlying the synergistic regulation of apical hook development by plant hormones.

\section{Results}

$\mathrm{GA}_{3}$ enhances while PAC represses ethylene- and EIN3induced hook curvature

Previous studies have shown that inhibition of GA biosynthesis by PAC prevents apical hook formation in both wild-type plants and the constitutive ethylene response mutant ctr1-1 [18]. To further reveal the molecular details of this effect, we examined the hook phenotypes in other ethylene mutants upon $\mathrm{GA}_{3}$ and/or PAC treatment. As shown in Figure 1A, PAC treatment inhibited the hook development of 3-day-old wild-type etiolated seedlings grown on Murashige and Skoog (MS) medium supplemented with or without ACC. In contrast, treatment with $\mathrm{GA}_{3}$ reversed the PAC effect, and the hook curvature was even more exaggerated in the presence of $\mathrm{ACC}+\mathrm{PAC}+\mathrm{GA}_{3}$ than that grown on $\mathrm{ACC}$ medium (Figure 1A). We further found that $\mathrm{GA}_{3}$ treatment showed a more pronounced effect to enhance ACCinduced hook curvature in 6-day-old etiolated seedlings, as seedlings grown on $\mathrm{ACC}+\mathrm{PAC}+\mathrm{GA}_{3}$ medium still exhibited exaggerated hook curvature, while only modest hook-bending was observed in seedlings grown on ACC 
A
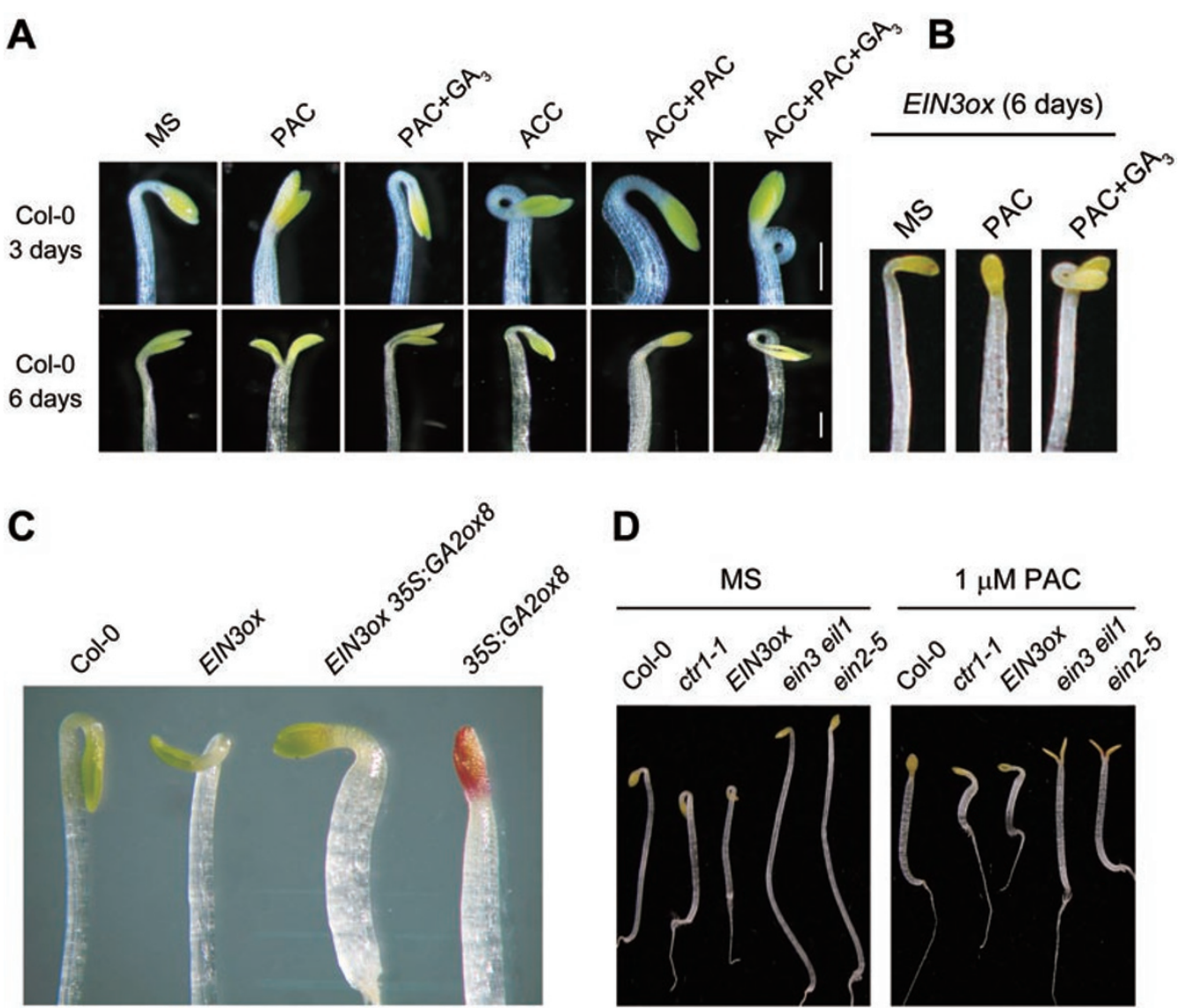

D
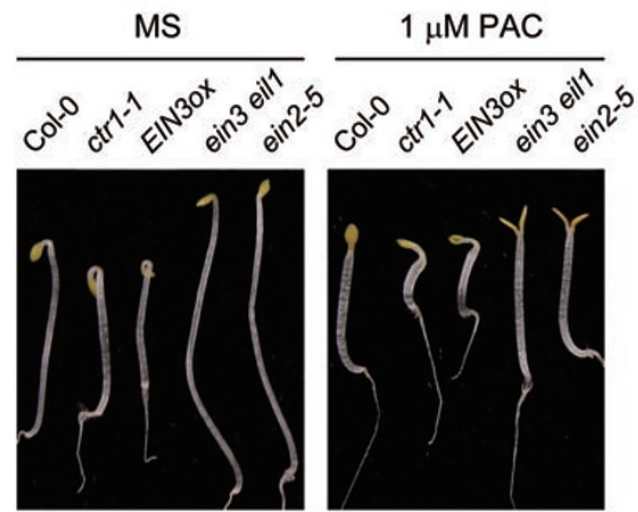

Figure 1 PAC represses while $\mathrm{GA}_{3}$ enhances the ethylene-induced hook curvature. (A) The hook phenotype of 3-day-old (top) and 6-day-old (bottom) etiolated wild-type seedlings grown on the indicated medium (1 $\mu \mathrm{M}$ PAC, $10 \mu \mathrm{M}$ ACC, and/or 10 $\mu \mathrm{M} \mathrm{GA} \mathrm{A}_{3}$ ). The scale bars represent $1 \mathrm{~mm}$. (B) The hook phenotype of 6-day-old etiolated seedlings of EIN3ox grown on the indicated medium (1 $\mu \mathrm{M}$ PAC and/or $10 \mu \mathrm{M} \mathrm{GA}$ ). (C) The hook phenotype of 3-day-old etiolated seedlings of indicated genotypes grown on MS medium. (D) The hook phenotype of etiolated ethylene-related mutants and transgenic plants grown on MS medium supplemented with or without $1 \mu \mathrm{M}$ PAC for 3 days.

or PAC $+\mathrm{GA}_{3}$ medium (Figure 1A).

EIN3/EIL1 are the central transcription factors in ethylene signaling [32], and overexpression of EIN3 or EIL1 results in constitutively exaggerated hook curvature $[33,34]$. We next sought to examine whether GA or PAC treatment affects the hook phenotype of EIN3 overexpression (EIN3ox). We found that PAC greatly suppressed the hook formation of 6-day-old EIN3ox seedlings, whereas $\mathrm{GA}_{3}$ reversed the PAC effect, and further exaggerated the hook bending (Figure 1B). Consistent with the results from exogenous PAC treatment, promotion of GA decay through overexpression of GA2ox8 $[35,36]$, which encodes a GA-inactivating enzyme (GA 2-oxidase), also repressed the hook curvature of EIN3ox (Figure 1C). These results indicate that application with GAs enhances while deprivation of GAs (by PAC treatment) represses EIN3-induced hook formation.
In addition, we found that upon PAC treatment, the 3-day-old seedlings with an activated ethylene signaling pathway (such as ctrl or EIN3ox) still showed bended hooks, whereas wild-type seedlings exhibited almost straight hooks, and ethylene-insensitive mutants (ein3 eill or ein2) were completely hookless with opened cotyledons (Figure 1D). These results suggest that seedlings with constitutively activated ethylene signaling are partially resistant to PAC's repression of hook bending, whereas ethylene-insensitive mutants are hypersensitive to the PAC effect.

della mutant exhibits exaggerated hook curvature that requires an intact ethylene signaling pathway

DELLA proteins are critical repressors of GA responses [37]. To further determine how GAs and PAC affect ethylene-induced hook curvature, we analyzed the 
hook phenotypes of various DELLA-related mutants and transgenic plants. The della quintuple mutant (with all five DELLA proteins mutated) [27] exhibited exaggerated apical hook on MS medium (Figure 2), which can be further enhanced by ACC treatment (Supplementary information, Figure S1). In contrast, transgenic plants overexpressing stabilized DELLA proteins (35S:TAPGAI 17 or 35S:TAP-RGAD17) [27] exhibited decreased hook curvature compared with wild type, and this was particularly true for 35S:TAP-RGA $\triangle 17$, which was almost hookless and evidently resistant to ACC treatment (Supplementary information, Figure S1). Together, these results suggest that DELLA proteins are repressors of ethylene-induced hook curvature, and that GA enhances ethylene action probably by destroying DELLA proteins.

To explore whether GA-enhanced hook curvature is caused by altered ethylene synthesis, della, penta (gal-3
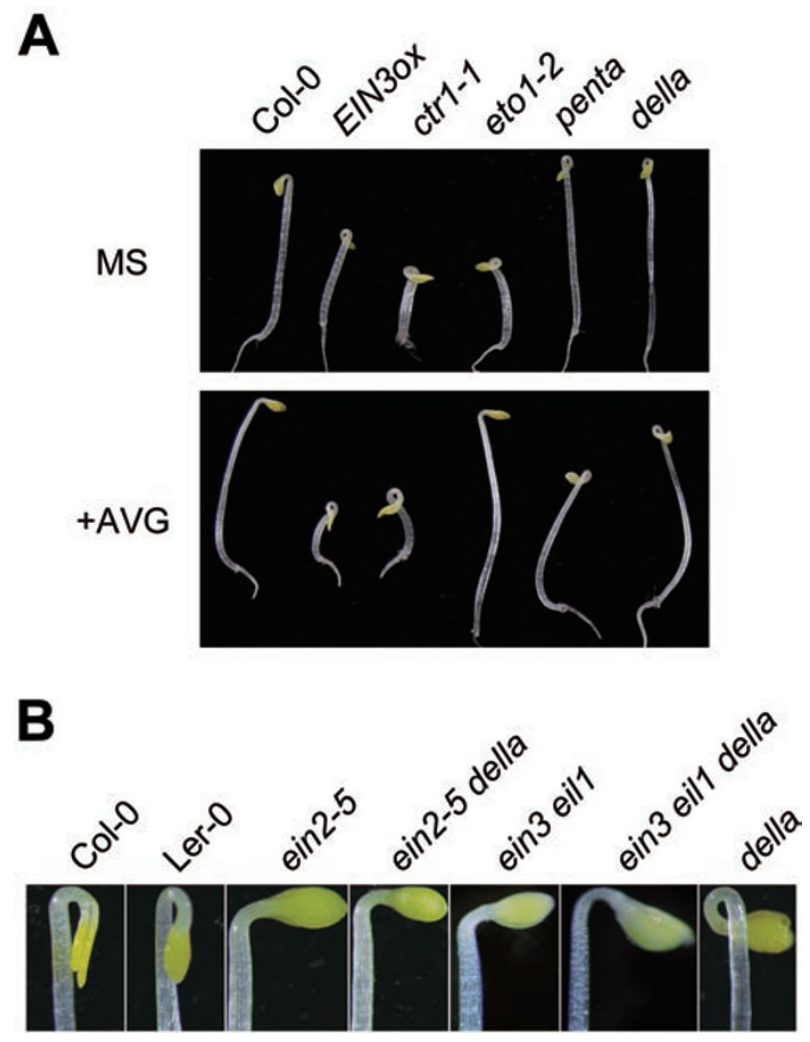

Figure 2 The della mutant exhibits exaggerated hook curvature that can be suppressed by ein2 or ein3 eil1. (A) The exaggerated hook curvatures of della (with all the five DELLA-mutated) and penta (ga1-3 gai-t6 rga-t2 rgl1-1 rgl2-1) were not affected by AVG (ethylene biosynthesis inhibitor) treatment. Etiolated seedlings were grown on MS medium supplemented with or without $10 \mu \mathrm{M}$ AVG for 3 days. (B) ein2 or ein 3 eil1 suppressed the exaggerated hook curvature of della. Etiolated seedlings of indicated genotypes were grown on MS medium for 3 days. gai-t6 rga-t2 rgll-1 rgl2-1), EIN3ox, ctr1, and ethyleneoverproduction mutants (etol) were grown on AVG medium, which blocks ethylene biosynthesis. As expected, AVG treatment repressed the constitutive hook curvature of etol (Figure 2A), an ethylene-overproduction mutant [12]. However, it had no effect on EIN3ox, ctr1, della, or penta mutant (Figure 2A). These results indicate that the exaggerated hook curvature of della is not caused by increased ethylene biosynthesis.

However, we found that genetic mutations in the downstream ethylene signaling components (such as ein2 and ein 3 eill) largely suppressed the exaggerated hookcurvature phenotype of della (Figure 2B). In addition, we observed that the della mutant exhibited an epinastic leaf phenotype, which results from exaggerated differential growth on the adaxial and abaxial sides of petioles [38]. Interestingly, the epinastic leaf phenotype of della was also suppressed by ein 2 or ein 3 eill (Supplementary information, Figure S2). Collectively, we conclude that EIN3/EIL1 are essential components required for the enhanced differential growth observed in della, including constitutive hook curvature and leaf epinastic growth.

\section{GA-enhanced hook curvature is HLS1 dependent}

HLS1 is essential for apical hook formation, and hls 1 fails to form a hook even in the presence of ACC $[8,12]$. In order to investigate whether HLS1 is involved in GA/ ethylene regulation of hook development, we observed the hook phenotype of $h l s 1$ under GA and/or ACC conditions and found that none of these treatments induced hook formation in hls 1 (Figure 3A). Furthermore, the exaggerated hook curvature of della was fully suppressed by $h l s 1$, as the hls 1 della mutant was also completely hookless (Figure 3B). These results suggest that GA- and DELLA-regulated hook development is dependent on HLS1.

GAs and ethylene induce HLS1 expression in an EIN3/ EIL1-dependent manner

Since ethylene likely augments hook curvature by inducing HLS1 expression, we asked whether GAs also regulate HLS1 expression as ethylene does. Given the nearly saturated GA response in dark-grown seedlings [39], wild-type seeds germinated in dark for $24 \mathrm{~h}$ were first transferred to $0.2 \mu \mathrm{M}$ PAC for $48 \mathrm{~h}$ to decrease the endogenous GA levels and then treated with 100 $\mu \mathrm{M} \mathrm{GA}_{3}$ for different time periods. We found that $\mathrm{GA}_{3}$ treatment evidently induced HLS1 expression after $2 \mathrm{~h}$ (Figure 4A). By demonstrating that the ethylene-induced expression of $H L S 1$ is EIN3/EIL1-dependent, as ACC treatment failed to induce $H L S 1$ expression in ein3 eill (Figure 4B), we asked whether the $\mathrm{GA}_{3}$-induced HLSI 
A

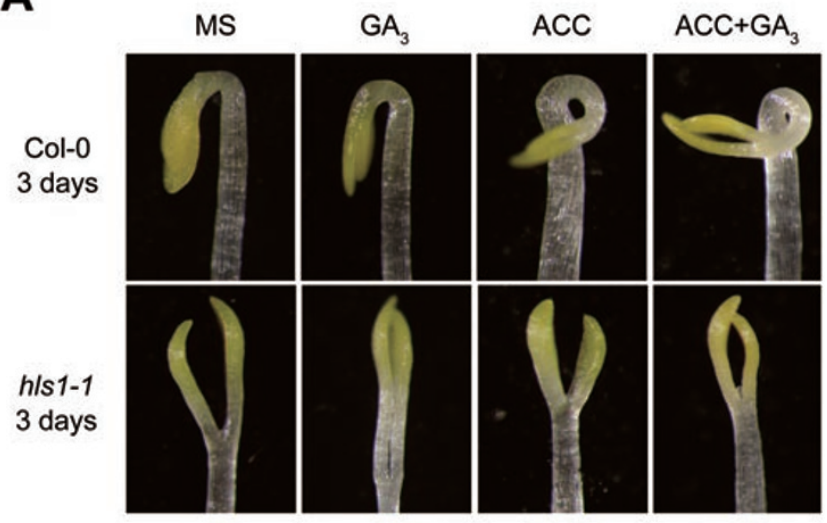

B

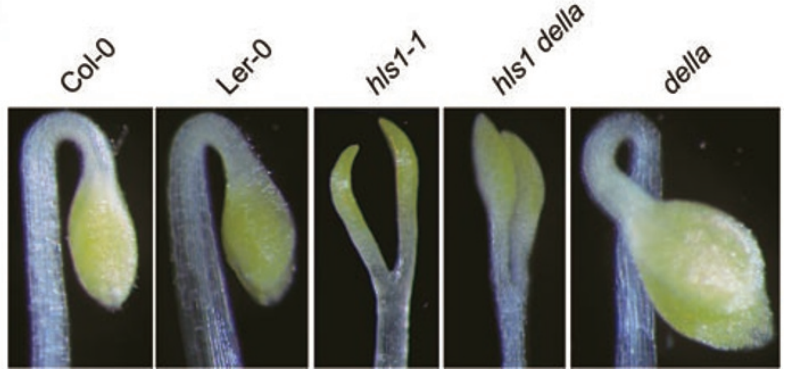

Figure 3 HLS1 is required for GA- and ethylene-induced hook curvature. (A) $\mathrm{GA}_{3}$ and/or ACC treatment did not affect the hookless phenotype of $h / s 1$. Hooks of 3-day-old etiolated seedlings grown on the indicated medium were shown. (B) h/s1 prevented the hook curvature of della. Hooks of 3-day-old etiolated seedlings grown MS medium were shown.

expression requires EIN3/EIL1. Our results revealed that $\mathrm{GA}_{3}$ also failed to induce $H L S 1$ expression in ein 3 eill (Figure 4A), indicating an essential role of EIN3/EIL1 in the HLS1 induction.

Next, we examined $H L S 1$ expression in various GArelated mutants. della mutants showed dramatically higher HLS1 expression than 35S:GA2ox8 transgenic plants (Figure 4C), which were presumed to accumulate less GAs and more DELLAs [36]. spy-3, another constitutive GA-response mutant with less DELLA accumulation [40], also showed higher HLS1 expression similar to della (Figure 4C). Furthermore, we found that overexpression of EIN3 in the ein3 eill background (EIN3ox/ein3 eil1) recovered the ethylene induction of HLS1 expression (Figure 4B), supporting that EIN3 is sufficient for HLS1 induction. Interestingly, manipulation of DELLA accumulation markedly influenced the effect of EIN3 on HLS1 induction, as 35S:GA2ox8 greatly inhibited while spy-3 evidently enhanced HLS1 expression in the EIN3ox background (Figure 4C). Collectively, these results demonstrate that ethylene and $\mathrm{GA}_{3}$ induce $H L S 1$ expression in an EIN3/EIL1-dependent manner.
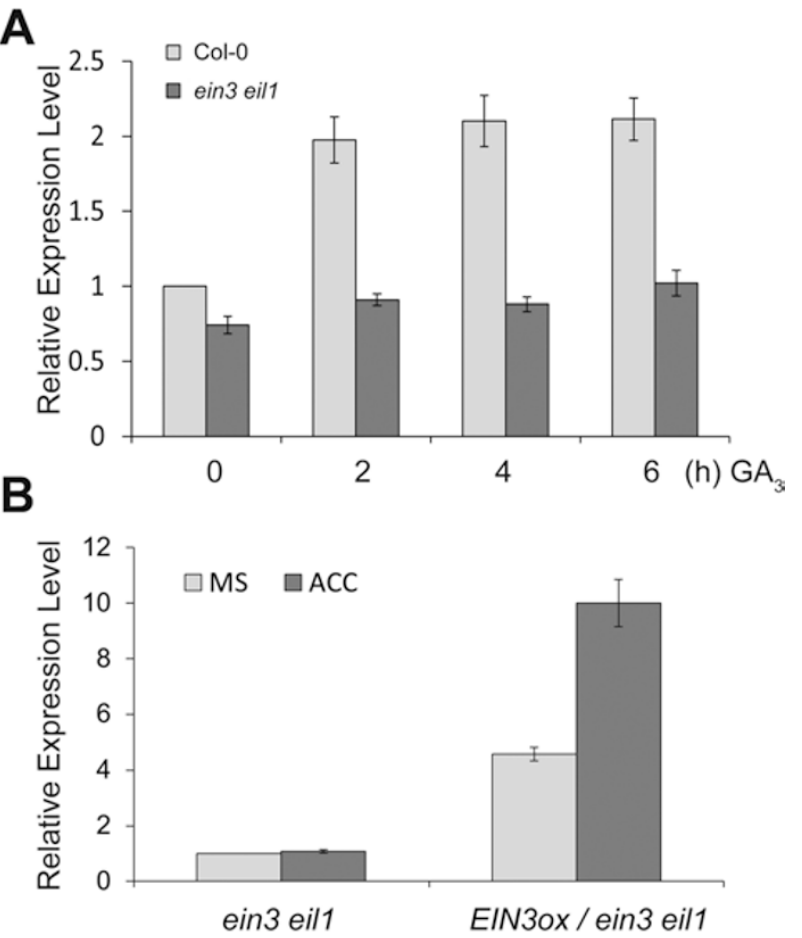

C

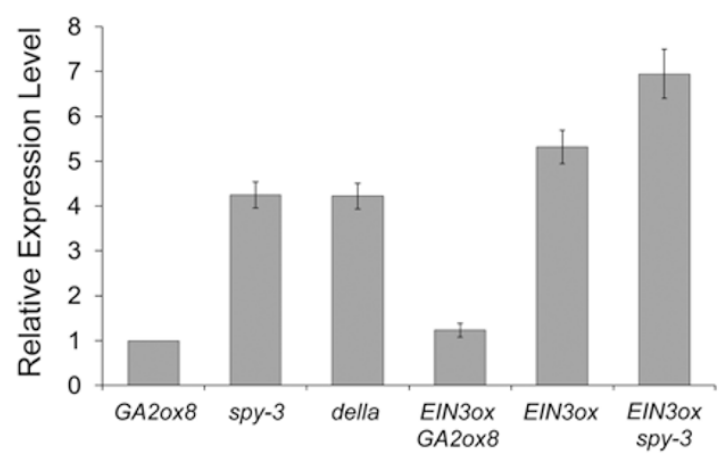

Figure 4 The $\mathrm{GA}_{3}$ - and ethylene-induced $H L S 1$ expression is EIN3/EIL1 dependent. (A) Quantitative reverse transcription PCR (qRT-PCR) analysis of the $\mathrm{GA}_{3}$-induced HLS1 expression in wild-type and ein 3 eil 1 seedlings. Seeds germinating in dark for $24 \mathrm{~h}$ were transferred to $0.2 \mu \mathrm{M} \mathrm{PAC}$ for $48 \mathrm{~h}$ and then treated with $100 \mu \mathrm{M} \mathrm{GA}_{3}$ for indicated hours prior to RNA extraction for qRT-PCR. (B) qRT-PCR analysis of the ethylene-induced HLS1 expression in ein3 eil1 and EIN3ox/ein3 eil1 (overexpression of EIN3 in ein3 eil1) seedlings. 3-day-old etiolated seedlings grown on MS medium were treated with or without $100 \mu \mathrm{M}$ ACC for $4 \mathrm{~h}$. (C) qRT-PCR analysis of HLS1 expression in the indicated genotypes. 3-day-old etiolated seedlings grown on MS medium were used for RNA extraction. The HLS1 expression levels in $\mathbf{A}, \mathbf{B}$, and $\mathbf{C}$ were normalized with the levels of $\beta$-tubulin. Mean $\pm \mathrm{SD}, n=3$. All experiments were repeated at least twice with similar results. 
EIN3 induces HLS1 expression by directly binding to its promoter

We next investigated how EIN3/EIL1 induces the expression of $H L S 1$. We generated a transgenic plant, which inducibly overexpressed EIN3-3FLAG in the ein3 eill background (iE/ein3 eil1). After induction with 10 $\mu \mathrm{M}$ estradiol, iE/ein3 eill displayed exaggerated hook curvature compared with ein 3 eill (Supplementary information, Figure S3), confirming the sufficiency of EIN3 for augmenting hook curvature. We further found that estradiol treatment rapidly induced HLS1 expression, within $1 \mathrm{~h}$ of EIN3 induction (Figure 5A), suggesting $H L S 1$ as a direct target gene of EIN3. A bioinformatic analysis found that a putative EIN3-binding site (EBS,
$5^{\prime}$-ATTTCAAA-3' [41]) is present in the $-900 \mathrm{bp}$ region upstream of the start codon of HLS1 (Figure 5B). To determine whether EIN3 directly binds to the putative EBS, we performed electrophoretic mobility shift assays (EMSAs) and found that EIN3 specifically bound to the wildtype EBS probe but not to a mutant probe (5'-CTGTAAGA-3') (Figure 5C). To further confirm the binding of EIN3 to the promoter sequences of HLS1, we conducted a chromatin immunoprecipitation (ChIP) assay in 3-dayold etiolated EIN3-FLAG/ein3 eil1 seedlings using antiFlag antibody. EIN3-FLAG/ein3 eill seedlings showed greatly increased binding of EIN3 to the EBS element compared with ein 3 eill seedlings. In contrast, a fragment from the $3^{\prime}$ untranslated region (UTR) of HLS1 did
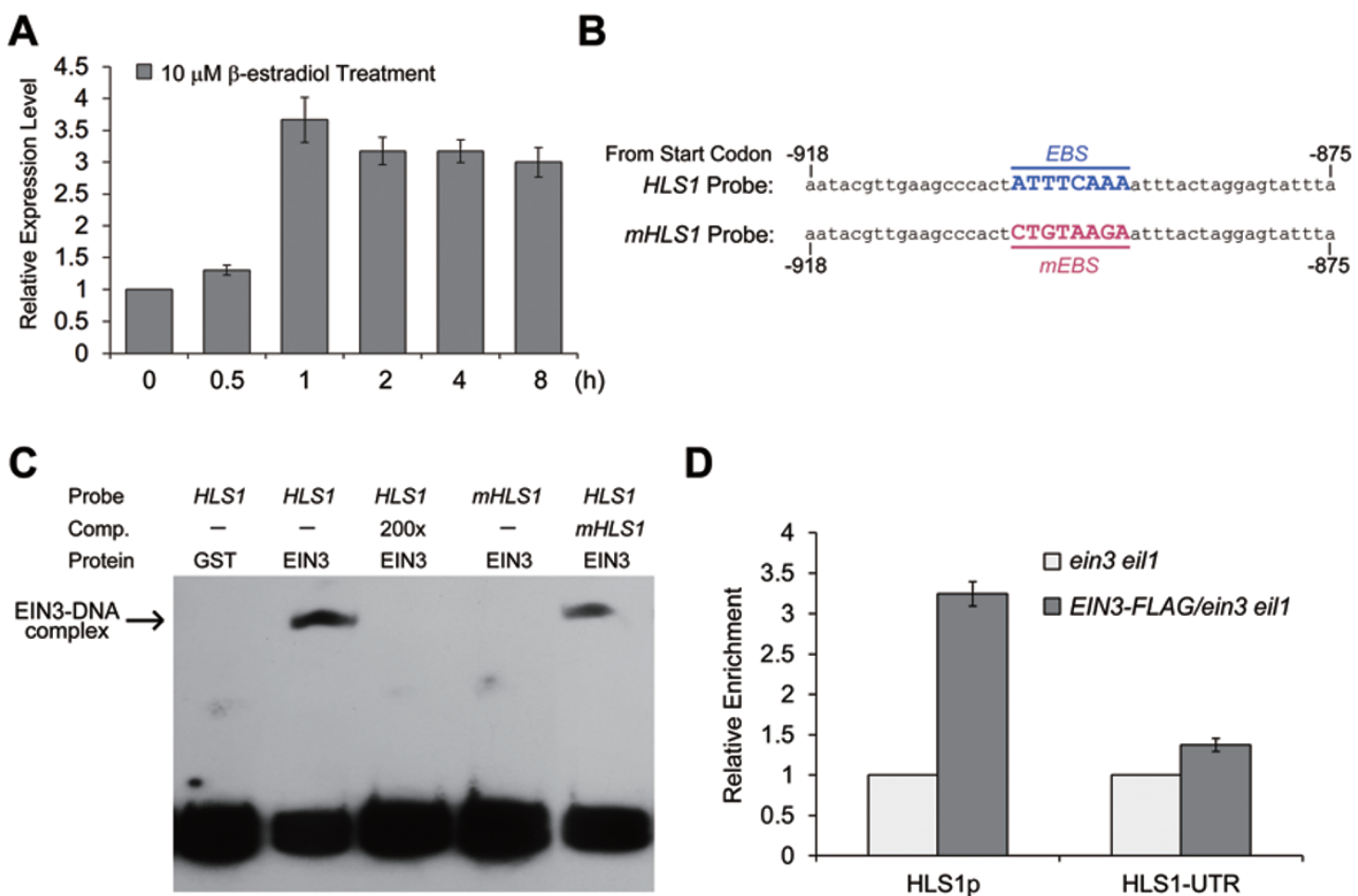

Figure 5 HLS1 is a direct target gene of EIN3. (A) Rapid induction of HLS1 expression in iE/ein3 eil1 by estradiol. 3-dayold etiolated iE/ein3 eil1 (a transgenic plant inducibly expressing EIN3-FLAG in the ein3 eil1 background) seedlings grown on MS medium were treated with $10 \mu \mathrm{M}$ estradiol for indicated hours before the seedlings were collected for RNA extraction. Experiments were repeated three times with similar results. (B) Oligonucleotides used in the EMSA assays. The HLS1 probe contains an EBS motif (underlined), which is a putative EBS. In the $m H L S 1$ probe, the EBS was mutated to $m E B S$ (underlined). Number showed the location upstream of the start codon. (C) EMSA results showing in vitro binding of GST-EIN3 to the EBS motif in the promoter of HLS1. Protein-DNA complexes were detected when GST-EIN3 was incubated with labeled HLS1 probe, and competition assays were conducted by adding 200 -fold excessive unlabeled HLS1 or $m$ HLS1 probe. GST-EIN3 was also incubated with labeled $m H L S 1$ probe and no protein-DNA complexes were observed. (D) ChIP assays indicating in vivo binding of EIN3 to the HLS1 promoter sequence. Chromatin from ein3 eil1 and transgenic plants constitutively expressing EIN3-FLAG in ein3 eil1 was immunoprecipitated with an anti-FLAG antibody, and the quantification of the indicated DNA fragments in the precipitated chromatin was determined by quantitative real-time PCR. The amounts of DNA amplified from the EIN3-FLAG/ein3 eil1 seedlings were normalized to that from ein3 eil1 plants. The 3'-UTR fragment of HLS1 was used as a negative control. The experiment was repeated three times with similar results. 
A

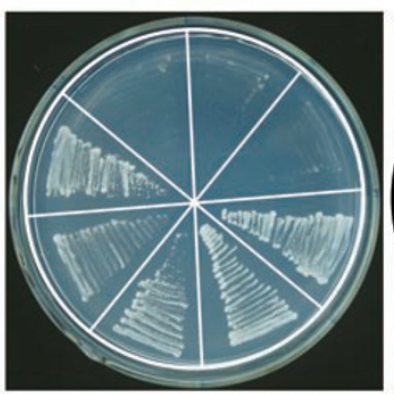

C
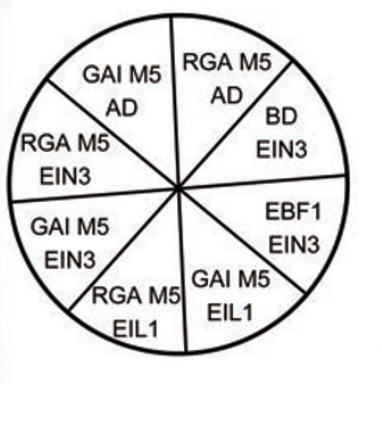

B

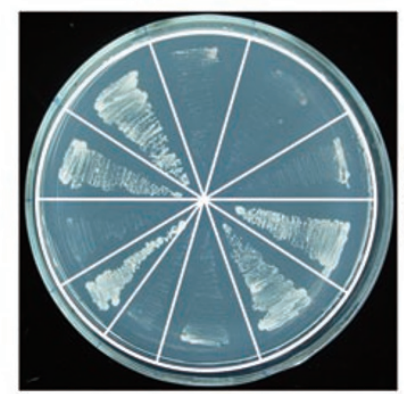

D

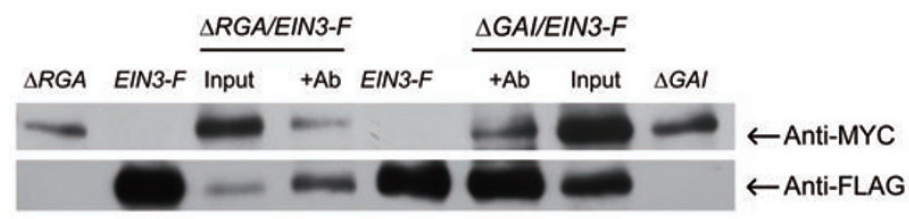

E
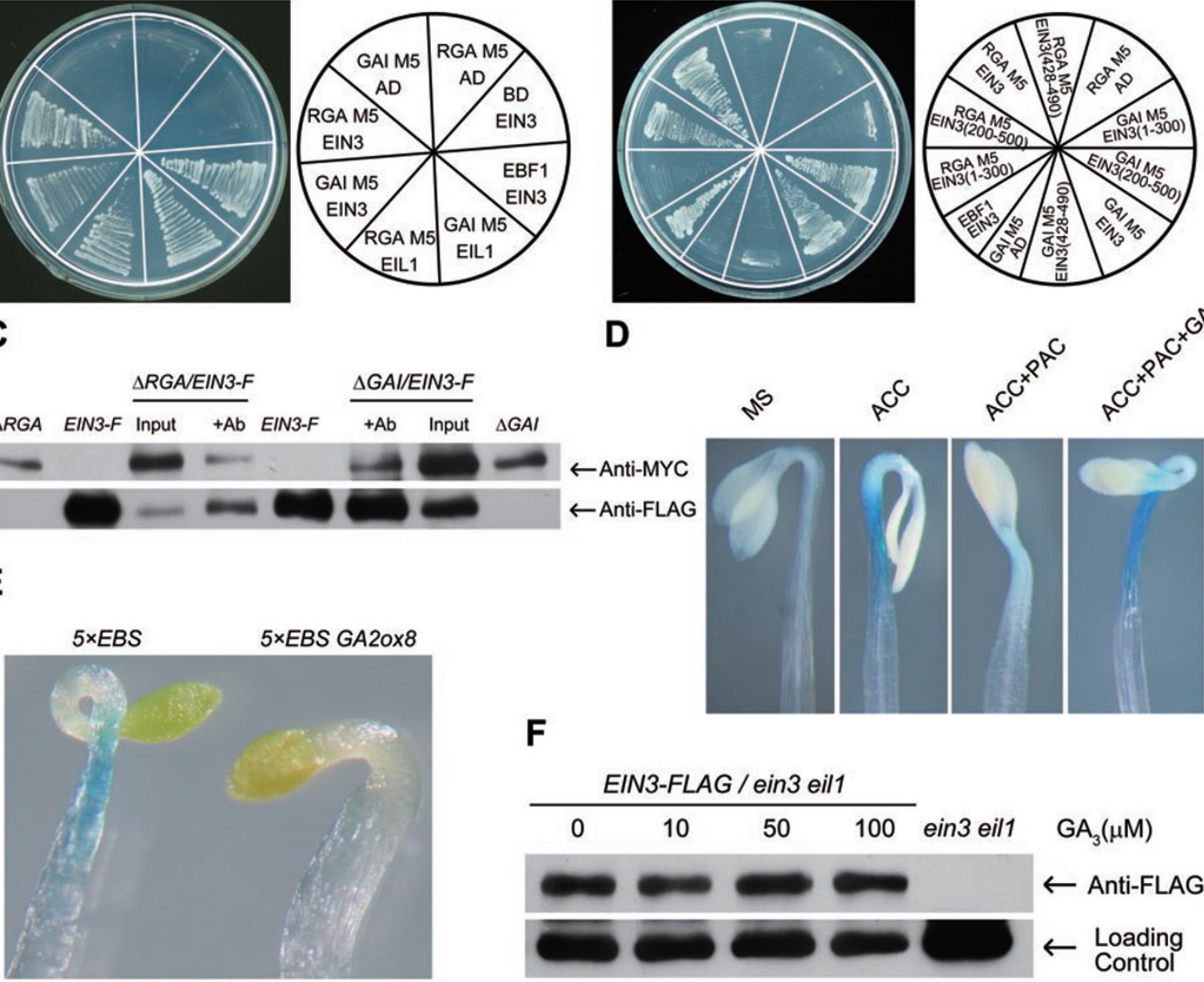

F
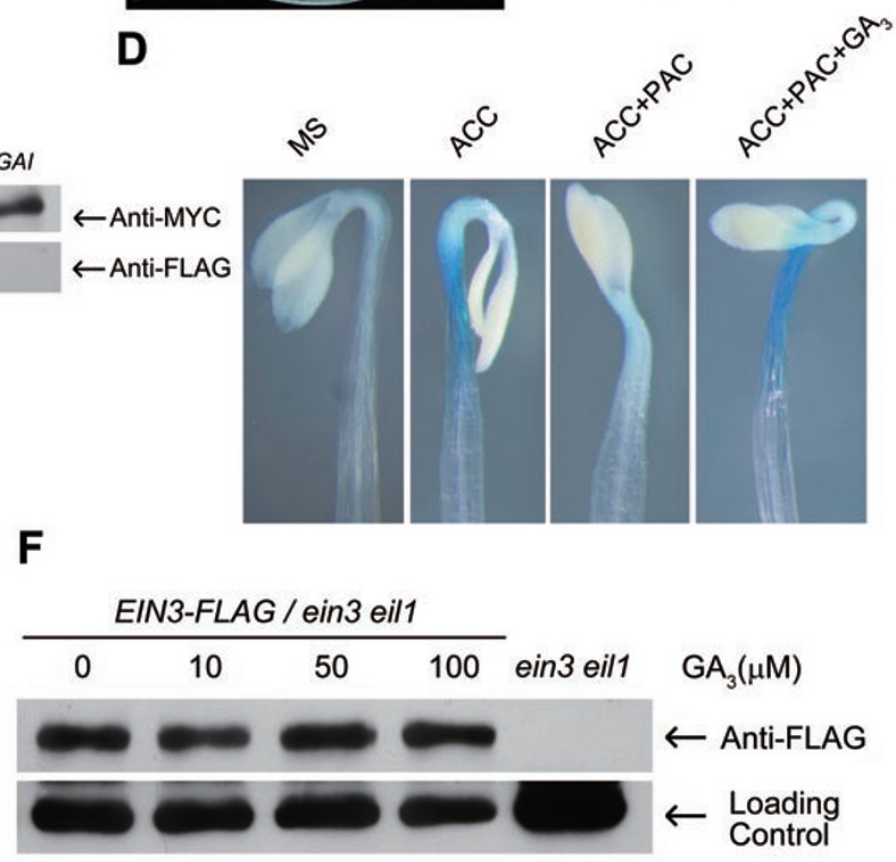

Figure 6 RGA/GAl interacts with EIN3/EIL1 and inhibits their function. (A) RGA/GAl interacted with EIN3/EIL1 in yeast cells. RGA M5 fragment (aa 209-587) and GAI M5 fragment (aa 158-533) were used in the yeast two-hybrid assays. EBF1/EIN3 interaction was used as a positive control. (B) EIN3 fragment (aa 200-500) was sufficient for the interactions with RGA and GAI in yeast cells. (C) Co-IP assays showing RGA/GAI association with EIN3 in vivo. EIN3-FLAG/ein3 eil1 (EIN3-F) was crossed with 35S:TAP-RGA $17(\triangle R G A)$ or 35S:TAP-GAI $17(\triangle G A I)$, and F1 plants were used for Co-IP. Proteins were immunoprecipitated with anti-FLAG M2 agarose beads (+Ab) and detected with either anti-MYC or anti-FLAG antibodies. (D) PAC treatment inhibited the ACC-induced 5×EBS:GUS expression. 3-day-old etiolated $5 \times E B S$ :GUS seedlings grown on the indicated medium (ACC: $10 \mu \mathrm{M}$, PAC: $1 \mu \mathrm{M}$, and GA: $10 \mu \mathrm{M}$ ) were used for GUS staining. (E) Expression of 35S:GA2ox8 in the 5×EBS:GUS background decreased the ACC-induced GUS staining. 3-day-old etiolated 5×EBS-GUS and 35S:GA20x8 $5 \times E B S$-GUS seedlings grown on $10 \mu \mathrm{M}$ ACC medium were used for GUS staining. $(F) G_{3}$ treatment did not affect the protein level of EIN3 in EIN3-FLAG/ein3 eil1. 3-day-old etiolated seedlings grown on $0.2 \mu \mathrm{M}$ PAC were treated with indicated concentrations of $\mathrm{GA}_{3}$ for $4 \mathrm{~h}$ before proteins were extracted for immunoblot assay with anti-FLAG antibody.

not show detectable enrichment by EIN3-FLAG (Figure 5D). Combining the results of in vitro EMSA and in vivo ChIP assays, we conclude that HLS1 is a direct target gene of EIN3.

RGA/GAI physically interacts with EIN3/EIL1 and inhibits their function

DELLA proteins are central transcriptional repressors without DNA-binding ability [26]. It has been reported that DELLAs associate with other transcription factors (like PIF3/4, ALC, and SCL3) to regulate downstream transcription and signal integration. Because DELLA proteins seemed to repress the expression of $H L S 1$, which is a direct target gene of EIN3/EIL1, we speculated that DELLAs could also repress EIN3/EIL1 functions through protein-protein interaction. We first examined the interactions between EIN3/EIL1 and RGA/GAI in yeast cells. To avoid the strong self-activation of full-length 

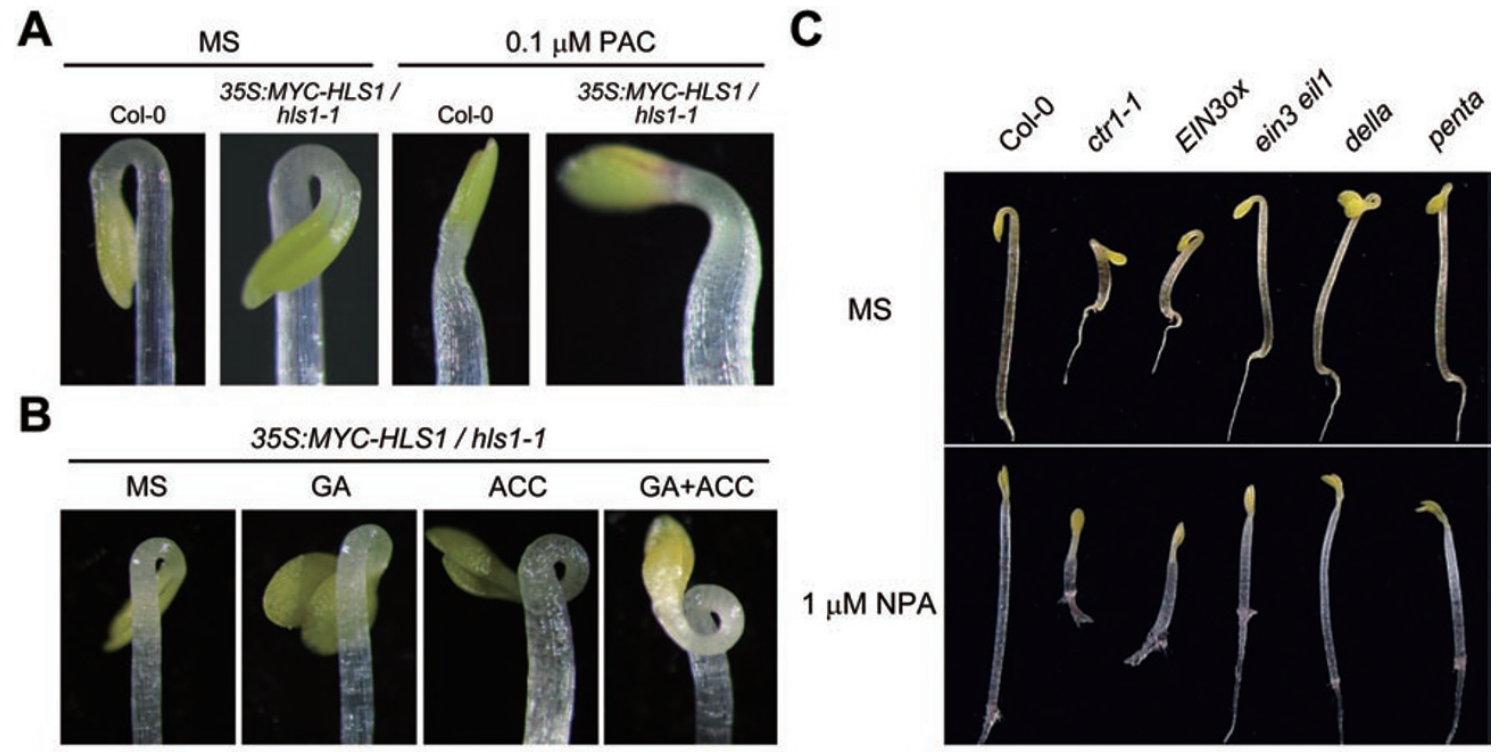

Figure 7 Blocking of auxin transport suppresses GA- and ethylene-induced hook curvature. (A) The hook phenotype of wildtype and 35S:MYC-HLS1/h/s1 grown on MS medium supplemented with or without $0.1 \mu \mathrm{M}$ PAC for 3 days. (B) The hook phenotype of 35S:MYC-HLS1/h/s1 grown on MS medium supplemented with or without $10 \mu \mathrm{M} \mathrm{ACC}$ and/or $10 \mu \mathrm{M}$ GA $\mathrm{A}_{3}$ for 3 days. (C) The hook phenotype of 3-day-old etiolated seedlings of indicated genotypes. Seedlings grown on MS medium and $1 \mu \mathrm{M}$ NPA medium were shown.

RGA or GAI [26], we chose the N-terminal fragments of RGA (M5: aa 209-587) or GAI (M5: aa 158-533) in our assays, and found that these fragments of RGA/GAI and EIN3/EIL1 exhibited interactions (Figure 6A). We also tested the interactions between other members of these two families, and no interactions were observed except for the EIL2 interaction with RGA (Supplementary information, Figure S4A). We further found that an EIN3 fragment containing amino-acid residues $200-500$ was sufficient for interacting with RGA/GAI (Figure 6B). Deletion of M5 from N-terminal or C-terminal disturbed the interaction (Supplementary information, Figure S4B). In addition, co-immunoprecipitation (Co-IP) studies using transgenic EIN3-3FLAG/ein3 eil1 crossed to 35S:TAP$R G A \Delta 17$ or $35 S: T A P-G A I \Delta 17$ further confirmed these interactions in planta (Figure 6C). Together, these results suggest that EIN3/EIL1/EIL2 are a new class of DELLAassociated transcription factors.

We next determined how RGA/GAI-EIN3/EIL1 interactions affect EIN3/EIL1 function. A $5 \times E B S: G U S$ reporter line harboring five tandem repeats of $E B S$ fused with the glucuronidase (GUS) reporter gene was previously used to monitor the function of EIN3/EIL1 [42]. We found that ACC evidently induced $5 \times \mathrm{EBS}$ :GUS expression in the hook/hypocotyl region, whereas the GUS expression was dramatically repressed by PAC treatment (Figure 6D). Moreover, $\mathrm{GA}_{3}$ reversed the PAC effect by enhancing the GUS expression. Consistently, overexpression of GA2ox 8 in the $5 \times E B S: G U S$ reporter line led to a reduction of ACC-induced GUS expression (Figure $6 \mathrm{E})$. These results suggest that accumulation of DELLA proteins acts to repress the function of EIN3/EIL1.

A principal mechanism for EIN3/EIL1 inactivation is to promote their protein degradation. We then tested whether GAs affect the protein level of EIN3, and found that $\mathrm{GA}_{3}$ treatments did not markedly affect EIN3 protein abundance in the EIN3-3FLAG/ein3 eill seedlings (Figure $6 \mathrm{~F})$. Since DELLA proteins interact with aa 200-500 of EIN3, a region responsible for DNA binding [43, 44], we speculate that DELLA/EIN3 interaction may repress the DNA-binding ability of EIN3.

Inhibiting auxin polar transport represses $G A$ - and ethylene-induced exaggerated hook curvature

Our above data suggest that GAs promote hook curvature probably by inducing $H L S 1$ expression. To further assess the importance of $H L S 1$ regulation in GA-regulated hook development, we overexpressed MYC-tagged HLS1 under the control of the constitutive $35 \mathrm{~S}$ promoter in hls1-1 background. As expected, the transgene expression rescued the hookless phenotype and the resulting plants exhibited exaggerated hook curvature even in the absence of ethylene or GAs (Figure 7A), supporting a role of HLS1 regulation in PAC-regulated hook curva- 
ture. We further found that 35S:MYC-HLS1/hls 1 was partially resistant to PAC treatment compared with wild type (Figure 7A). However, 35S:MYC-HLSl/hls 1 was still responsive to $\mathrm{PAC}, \mathrm{ACC}$, and $\mathrm{ACC}+\mathrm{GA}_{3}$ (Figure 7B), we thus examined the mRNA and protein levels of HLS1 in 35S:MYC-HLS1/hls 1, and found that neither mRNA level nor protein level of MYC-HLS1 was altered by $\mathrm{ACC}$ or $\mathrm{GA}_{3}$ treatment (Supplementary information, Figure S5), indicating that there must be alternative pathways independent of $H L S 1$ transcription to mediate the effects of GAs and PAC on hook-curvature regulation. Previous studies have shown that treatment with NPA prevented the constitutive hook curvature of HLSIox [8]. We next examined whether NPA also suppresses the exaggerated hook phenotypes caused by the activation of either ethylene or GA signaling. We found that treatment with NPA almost completely inhibited the apical hook-bending observed in ctr1, EIN3ox, della, and penta mutants (Figure 7C). This result indicates that auxin functions downstream of the ethylene and GA pathways to modulate hook curvature, thus raising a possibility that GAs and ethylene also influence auxin transport in addition to inducing HLS1 expression. This conclusion is consistent with a recent report showing that GAs modulate auxin transport and response through PIN3 and PIN7 [45].

\section{Discussion}

Apical hook is a unique functional structure essential for plant germination in the soil that protects the cotyledons and apical meristemic stem from mechanical damage when protruding through the soil. Its formation is coordinately regulated by several plant hormones, including ethylene, auxin, GAs, jasmonate, and brassinosteroid $[3,16-18,46-48]$. The interplay between ethylene and auxin was extensively studied and several regulatory mechanisms have been revealed. However, the molecular details of the interactions among other hormones in the control of hook development remain largely unknown. In this study, we show that GAs and ethylene cooperatively regulate the hook curvature partly by inducing the gene expression of HLS1, a putative $N$-acetyltransferase essential for hook formation. We further demonstrate that EIN3/EIL1s are the integration node linking the two hormone pathways to directly activate $H L S 1$ transcription, and that GAs induce HLS1 expression by relieving the inhibitory effect of DELLA proteins on EIN3/EIL1. In the meanwhile, GAs and ethylene also initiate the HLS1independent pathways to regulate hook curvature, probably by modulating asymmetric auxin accumulation in the hook region (Figure 8).

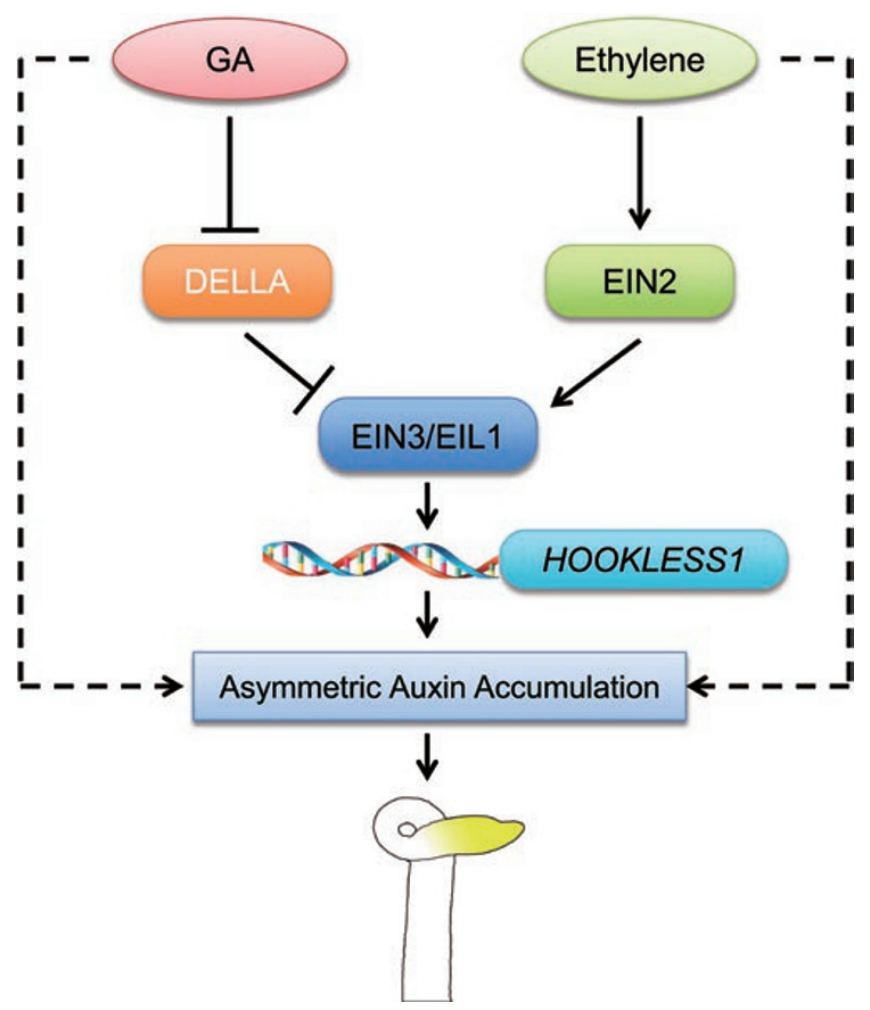

Figure 8 A proposed model of GAs and ethylene co-action in promoting the hook curvature. GAs and ethylene cooperatively regulate the hook curvature partly by inducing the gene expression of $H L S 1$, a putative $N$-acetyltransferase essential for hook formation. EIN3/EIL1 are the integration node linking the two hormone pathways to directly activate the HLS1 transcription, in which ethylene stabilizes EIN3/EIL1, while GAs relieve the repression of DELLA proteins on EIN3/EIL1. Meanwhile, GAs and ethylene also initiate the HLS1-independent pathways to regulate hook curvature, probably by modulating asymmetric auxin accumulation in the hook region. The solid lines indicate proved regulations, whereas the dotted lines indicate proposed regulations.

Treatment of PAC, a GA-biosynthesis inhibitor, was previously reported to radically repress ethylene-induced hook curvature. Our study provides convincing evidence to indicate that PAC/GA-mediated regulation of hook curvature is partially dependent on the action of EIN3/ EIL1, two ethylene-stabilized transcription factors. First, we found that overexpression of EIN3 (EIN3ox) resulted in partial resistance to either PAC treatment (Figure 1D) or GA deficiency (Figure 1C) in terms of hook curvature. EIN3ox also exhibited exaggerated hook bending upon $\mathrm{GA}_{3}$ treatment, reminiscent of wild-type seedlings treated with ethylene plus $\mathrm{GA}_{3}$ (Figure 1A and 1B). Second, loss of EIN3/EIL1 function remarkably suppressed the constitutive hook-bending phenotype of della mutant 
(Figure 2). Similarly, della mutant was also suppressed by ein2, a mutant with no EIN3 or EIL1 protein accumulation. Third, we showed that HLS1 is a direct target gene of EIN3 (Figure 5), and further found that $\mathrm{GA}_{3}$-induced HLS1 expression was dependent on EIN3/EIL1 (Figure 4). Lastly, we revealed that both EIN3 and EIL1 physically interacted with DELLA proteins, and such association led to the inhibition of EIN3/EIL1-regulated gene expression (Figure 6).

On the other hand, it is notable that GA control of hook curvature is not solely dependent on the EIN3/EIL1 pathway, as the ein 3 eill mutant was still responsive to PAC treatment (Figure 1D). In addition, ein3 eill suppressed the constitutive hook phenotype of della to a half way (Figure 2B), whereas NPA treatment almost completely blocked its hook formation (Figure 7C). Therefore, DELLA proteins might direct an additional pathway independent of EIN3/EIL1 to modulate auxin production, transport, or signaling in hook development. DELLA proteins were reported to interact with PIF3/4 transcription factors and repress their activity. Interestingly, loss of multiple PIF functions also resulted in a hookless phenotype [49], suggesting an important role of PIF proteins in maintaining hook curvature. Together, these results indicate that GA-increased hook curvature is mediated through the removal of DELLA proteins, and DELLA accumulation reduces hook curvature by repressing the functions of EIN3/EIL1, and probably PIFs as well, to modulate the downstream auxin responses.

Previous studies have demonstrated that HLS1 is a key mediator of multiple signals, including ethylene, auxin, and light, in the control of hook curvature [3]. Our results further support the central role of HLS1 in mediating hormone signals (GAs and ethylene) in hook development. We first showed that the hls 1 mutant was completely insensitive to ethylene and/or $\mathrm{GA}_{3}$ treatment (Figure 3A), and the mutation also suppressed the exaggerated hook phenotype of the della mutant (Figure 3B). Furthermore, we found that both $\mathrm{GA}_{3}$ and ethylene induced HLS1 expression (Figure 4), and that EIN3 appears to directly bind to the promoter of HLS1 (Figure 5). Thus, induction of HLS1 expression represents a principal mechanism of GA- and ethylene-mediated regulation of hook development. Nevertheless, we also observed that plants with constitutive overexpression of HLS1 in the hls 1 -mutant background (HLS1ox/hls 1$)$ were still responsive to ethylene, $\mathrm{PAC}, \mathrm{GA}_{3}$, or their combined treatment (Figure 7A and 7B). Given that the transcript or protein level of MYC-HLS1 was not affected by ACC and $\mathrm{GA}_{3}$ (Supplementary information, Figure S5), these results suggest the existence of the alternative GA and ethylene signaling pathways independent of HLS1 induc- tion (Figure 8). Our results and previous studies showed that blocking of auxin transport by NPA represses the constitutive hook curvature of HLS1ox, ctr1, EIN3ox, and della (Figure 7C), suggesting that modulation of auxin distribution is a common downstream event in the GA- and ethylene-mediated regulation of hook formation. Ethylene was also recently reported to regulate hook curvature by influencing auxin influx and efflux carriers at transcriptional and posttranscriptional levels $[16,17]$. It is thus possible that GAs might alter the expression level or activity of auxin influx and/or efflux transporters as well.

Accumulating evidence has demonstrated that DELLA proteins control a wide variety of plant growth and stress responses. With no intrinsic DNA-binding abilities, DELLA proteins were thought to function as transcriptional repressors that interact with various transcription factors and repress their activity [24, 25]. To date, DELLA proteins have been reported to interact with PIF3/4, ALC, and SCL3 transcription factors to mediate the effects of GA on hypocotyl elongation, valve margin development, and root development, respectively. In this study, we have identified EIN3/EIL1 as a new class of DELLA-associated transcription factors. Interestingly, only RGA and GAI but not RGL proteins interacted with EIN3/EIL1, suggesting the functional diversity of DELLA proteins in different processes. As $\mathrm{GA}_{3}$ treatment had no effect on EIN3 protein stability, plus the observation that DELLA proteins interacted with the DNA-binding domains of EIN3/EIL1, we speculate that DELLA association could block the binding of EIN3/EIL1 to their target promoters. Further experiments would help verify this possibility in the future. Since GAs and ethylene coordinately regulate a number of plant growth and development processes, such as seed germination, stem elongation, and stress tolerance, it would be interesting to investigate whether the DELLA-EIN3/EIL1 interaction also plays a part in the coordinated action of the two hormones in these processes.

\section{Materials and Methods}

\section{Plant materials and growth conditions}

ein2-5 [50], ein3-1 eill-1 [32], ctr1-1 [14], etol-2 [12], hls1-1 [12], penta (gal-3 gai-t6 rga-t2 rgll-1 rgl2-1) [51], and della (rgat2 gai-t6 rgll-1 rgl2-1 rgl3-1) [27] mutants and EIN3ox (35S:EIN3) [34], iE/qm [33], $5 \times E B S: G U S$ [42], 35S:TAP-RGA $17(\triangle R G A)$ [27], 35S:TAP-GAIAI7( $\triangle G A I)$ [27], and 35S:GA2ox8 [35] transgenic plants were described previously.

della hls1, della ein2, and della ein 3 eill multiple mutants were generated by genetic crosses, and putative etiolated della hls 1 , della ein 2 , and della ein 3 eill homozygous lines were initially identified by comparison with the parental phenotype on the MS medium supplemented with $10 \mu \mathrm{M}$ PAC plus $10 \mu \mathrm{M} \mathrm{ACC}$, then 
confirmed through PCR-based genotyping. The primer pairs for identifying ein2, ein3, eil1, and DELLA-related mutants were as described previously [33, 52].

Estradiol-inducible EIN3-FLAG in the ein3 eill background (iE/ein3 eill) was generated by crossing $i E / q m$ (estradiol-inducible EIN3-FLAG in the ein3 eil1 ebf1 ebf2 background) into ein3 eil1 background and then screened on $10 \mu \mathrm{M}$ estradiol in the dark. Those with longer roots compared with $i E / q m$ were further genotyped with the $e b f 1$ and $e b f 2$ sites. The primer pairs for identifying $e b f 1$ and $e b f 2$ were as described previously [33].

To construct transgenic plants constitutively expressing EIN3-3FLAG, EIN3 cDNA was PCR amplified from reverse transcription product with primers 5'-AGGTTCGAAGAACCATATGGATACATCTTG3'and 5'-AAACTCGAGATGATGTTTAATGAGATGGGAATG-3', digested with $X h o \mathrm{I}$ and $\operatorname{Csp} 45 \mathrm{I}$, inserted into a pUC19-derived plasmid containing triple FLAG-tag, and then digested with KpnI and Pst I, inserted into a pCAMBIA1300 vector. To construct transgenic plants constitutively expressing MYC-HLS1, HLS1 cDNA was PCR amplified from reverse transcription product with primers 5'-TCCCCCGGGACATGACGGTGGTTAGAGAGTAC-3' and 5'-GCGGAGCTCGTAGGAGATATTACCTCTTACC3-', digested with $S m a \mathrm{I}$ and $S a c \mathrm{I}$, inserted into a pBA-MYC vector. All constructs were verified by DNA sequencing analysis. The resultant constructs were introduced into Agrobacterium strain C58, and subsequently the $35 S$ :EIN3-3FLAG construct was transformed into ein3-1 eil1-1 plants and the 35S:6MYC-HLS1 constructs were transformed into $h l s 1-1$ by vacuum infiltration [53].

Surface-sterilized seeds were plated on MS medium $(4.3 \mathrm{~g} / 1$ MS salts, $1 \%$ sucrose, $\mathrm{pH} 5.7-5.8$, and $8 \mathrm{~g} / \mathrm{l}$ agar) and imbibed for 4 days at $4{ }^{\circ} \mathrm{C}$. For hook phenotype, the plates were wrapped in foil and kept in a $22{ }^{\circ} \mathrm{C}$ incubator before the phenotypes of seedlings were analyzed. For adult plant phenotypic analysis, lightgrown seedlings from plates were transferred to soil and grown to maturity at $22^{\circ} \mathrm{C}$ under a 16 -h light/8-h dark cycle.

\section{Chemical solutions}

All chemicals were from Sigma-Aldrich, unless specified otherwise, and prepared as stock solutions. DMSO was used to dissolve NPA $(100 \mu \mathrm{M}), \mathrm{MG} 132(50 \mathrm{mM})$, and $\beta$-estradiol $(10 \mathrm{mM}) .100 \%$ ethanol was used to dissolve $\mathrm{GA}_{3}(71.43 \mathrm{mM})$ and PAC $(10 \mathrm{mM})$. ACC $(10 \mathrm{mM})$ and AVG $(10 \mathrm{mM})$ were dissolved in water. Final concentrations were $1 \mu \mathrm{M}$ NPA, $10 \mu \mathrm{M}$ ACC, and $10 \mu \mathrm{M}$ AVG for germination, $50 \mu \mathrm{M}$ MG132 for Co-IP, and $100 \mu \mathrm{M} \mathrm{GA}$ and 100 $\mu \mathrm{M}$ ACC for seedling treatment.

\section{Immunoblot assays}

For EIN3-3FLAG western blot, 3-day-old dark-grown EIN3:3FLAG/ein3 eil1 transgenic seedlings were transferred to MS liquid medium with different concentrations of $\mathrm{GA}_{3}$ treatment for $4 \mathrm{~h}$ and protein was extracted in the buffer $(50 \mathrm{mM}$ Tris-Cl, pH 7.5, $150 \mathrm{mM} \mathrm{NaCl}, 1 \mathrm{mM}$ EDTA, 0.25\% Triton X-100, $1 \mathrm{mM}$ PMSF, and $1 \times$ protease inhibitor cocktail). EIN3-3FLAG fusion proteins were determined by immunoblot using anti-FLAG antibody (Sigma-Aldrich) diluted in 5000 folds.

\section{Real-time quantitative reverse transcription PCR analysis of gene expression \\ Total RNA was prepared using the TRIzol Reagent (Invitrogen, Inc.). In all, $2 \mu \mathrm{g}$ total RNA treated with DNase I (Promega, Inc.)}

was added in a $20 \mu \mathrm{l}$ reverse transcription reaction using the MMLV Reverse Transcription System (Promega, Inc.). Real-time PCR was performed on the LightCycler 480 Real-Time PCR System (Roche) using SYBR Green Mix (Takara, Inc.) and the specific primers of HLS1 (5'-CACGGTTATCAAGTTAGAGC-3' and 5'-GAAAGTCCCAAGCGAGA-3'). The expression level was normalized to $\beta$-tubulin (5'-GAGCCTTACAACGCTACTCTGTCTGTC-3' and 5'-ACACCAGACATAGTAGCAGAAATCAAG-3') control. Experiments were biologically repeated at least two times.

\section{ChIP assay}

ChIP was performed as described [43] using 3-day-old darkgrown seedlings on MS medium treated with $100 \mu \mathrm{M} \mathrm{ACC}$. Mouse anti-FLAG M2-agarose beads (Sigma-Aldrich) were used for IP. The enrichment of DNA fragments was determined by quantitative real-time PCR using primer pairs HLS1-pchip: 5'-CCACATCAATGCTCGTCTTA-3' and HLS1-pr-chip: 5'-CAGTGGCGCTATCTATTTTC-3', and primer pairs: HLS1-3' UTR-CHIP: 5'-GGCGTTTCCATTTTTGTAGAC-3', and HLS1-3' UTRR-CHIP: 5'-GGAGATATTACCTCTTACCAC-3' were used as a negative control.

\section{Protein expression}

GST-EIN3 (aa 1-314) constructs were transformed into BL21 (DE3)-competent cells. Protein expression was induced by 0.1 $\mathrm{mM}$ isopropyl-beta-thiogalactopyranoside and fusion proteins were prepared following the manufacturer's instructions.

\section{EMSA}

Oligonucleotide probes (HLS1: 5'-AATACGTTGAAGCCCACTATTTCAAAATTTACTAGGAGTATTTA-3' and 5'-TAAATACTCCTAGTAAATTTTGAAATAGTGGGCTTCAACGTATT-3'; mHLS 1: 5'-AATACGTTGAAGCCCACTCTGTAAGAATTTACTAGGAGTATT-3' and 5'-AATACTCCTAGTAAATTCTTACAGAGTGGGCTTCAACGTATT-3') were synthesized and labeled with a Biotin 3'-end DNA Labeling Kit. EMSA was performed using a LightShift Chemiluminescent EMSA Kit. In total, $20 \mathrm{fmol}$ of labeled probes were incubated in $1 \times$ binding buffer, $2.5 \%$ glycerol, $50 \mathrm{mM} \mathrm{KCl}, 5 \mathrm{mM} \mathrm{MgCl}_{2}$, and $10 \mathrm{mM}$ EDTA with or without proteins at room temperature for 20 min. For non-labeled probe competition, the amount of HLS1 and mHLS1 competitors is 200 -fold more than that of labeled probe.

\section{Yeast two-hybrid assays}

The coding sequences of RGA M5 (209-587), RGA (256-587), $R G A$ (323-587), RGA (209-372), RGA (209-294), GAI M5 (158533), RGL1 M5, RGL2 M5, and RGL3 M5 were amplified from wild-type cDNA and cloned into pGBKT7 vectors, while the coding sequences of EBF1, EIN3, EIN3 (200-500), EIN3 (1-300), EIL1, EIL2, EIL3, EIL4, and EIL5 were cloned into pGADT7 vectors and transformed into yeast strain AH109 following the Matchmaker user's manual protocol (Clontech). Transformed yeast cells were streaked onto SD (-Leu/-His/-Ade/-Trp) medium (Clontech) and grown at $30{ }^{\circ} \mathrm{C}$ for 4 days. The white colonies represented interactions. All the primers used in this study are summarized in Supplementary information, Table S1.

\section{EIN3-3FLAG and $\triangle R G A-M Y C / \triangle G A I-M Y C$ Co-IP assays}

Co-IP assays of the EIN3-RGA/GAI proteins were performed 
with 7-day-old $\mathrm{F} 1$ seedlings derived from genetic crosses between EIN3-3FLAG/ein3 eil1 (EIN3-F) and $\triangle R G A-M Y C / \triangle G A I-M Y C$ grown under white light. Seedlings were treated with $100 \mu \mathrm{M}$ $\mathrm{ACC}$ for $4 \mathrm{~h}$ prior to protein extraction. Immunoprecipitation of the EIN3-3FLAG protein was performed at $4{ }^{\circ} \mathrm{C}$ for at least $6 \mathrm{~h}$, using anti-FLAG M2-agarose beads (Sigma-Aldrich) in a buffer containing $50 \mathrm{mM}$ Tris-Hcl, pH 7.5, $150 \mathrm{mM} \mathrm{NaCl}, 1 \mathrm{mM}$ EDTA, $0.25 \%$ Triton X-100, $1 \mathrm{mM}$ PMSF, and $1 \times$ protease inhibitor cocktail (Roche). EIN3-3FLAG and $\triangle$ RGA-MYC/DGAI-MYC detection were performed with anti-FLAG and anti-MYC antibodies, respectively.

\section{GUS staining}

Seedlings were incubated in GUS staining buffer $(100 \mathrm{mM}$ $\mathrm{Na}_{3} \mathrm{PO}_{4}, \mathrm{pH} 7.0,1 \mathrm{mM}$ EDTA, $1 \mathrm{mM}$ potassium ferrocyanide, 1 $\mathrm{mM}$ potassium ferricyanide, $1 \%$ Triton $\mathrm{X}-100$, and $1 \mathrm{mg} / \mathrm{ml} \mathrm{X-}$ Gluc) and then washed in washing solution $\left(100 \mathrm{mM} \mathrm{Na}_{3} \mathrm{PO}_{4}\right.$ and $1 \mathrm{mM}$ EDTA) before images were taken.

\section{Acknowledgments}

We thank Dr Xingwang Deng (Yale University, USA) for providing 35S:TAP-RGAD17, 35S:TAP-GAIA17, and della seeds, Dr Chentao Lin (University of California, Los Angeles, USA) for providing 35S:GA20x8 seeds, and Dr Xiangdong Fu (Institute of Genetics and Developmental Biology, Chinese Academy of Sciences, China) for providing spy-3 and penta seeds. This work was supported by the National Natural Science Foundation of China (91017010 and 30730011 to HG and 30800078 to FA) and the Ministry of Science and Technology of China (2009CB119101 to HG). The publication fee is covered by the 111 Project of Peking University.

\section{References}

1 Darwin C, Darwin F. The power of movement in plants. New York: D Appleton and Co, 1881:87-94.

2 Silk WK, Erickson RO. Kinematics of hypocotyl curvature. Am J Bot 1978; 65:310-319.

3 Li H, Johnson P, Stepanova A, Alonso JM, Ecker JR. Convergence of signaling pathways in the control of differential cell growth in Arabidopsis. Dev Cell 2004; 7:193-204.

4 Schwark A, Schierle J. Interaction of ethylene and auxin in the regulation of hook growth I: the role of auxin in different growing regions of the hypocotyl hook of Phaseolus vulgaris. J Plant Physiol 1992; 140:562-570.

5 Boerjan W, Cervera MT, Delarue M, et al. Superroot, a recessive mutation in Arabidopsis, confers auxin overproduction. Plant Cell 1995; 7:1405-1419.

6 Zhao Y, Christensen SK, Fankhauser C, et al. A role for flavin monooxygenase-like enzymes in auxin biosynthesis. Science 2001; 291:306-309.

7 Stepanova AN, Robertson-Hoyt J, Yun J, et al. TAA1-mediated auxin biosynthesis is essential for hormone crosstalk and plant development. Cell 2008; 133:177-191.

8 Lehman A, Black R, Ecker JR. HOOKLESS1, an ethylene response gene, is required for differential cell elongation in the Arabidopsis hypocotyl. Cell 1996; 85:183-194.

9 Abeles FB, Morgan PW, Saltveit JME. Ethylene in Plant Biol- ogy. 2nd Edition. San Diego: Academic Press, 1992.

10 Ecker JR. The ethylene signal transduction pathway in plants. Science 1995; 268:667-675.

11 Bleecker AB, Estelle MA, Somerville C, Kende H. Insensitivity to ethylene conferred by a dominant mutation in Arabidopsis thaliana. Science 1988; 241:1086-1089.

12 Guzman P, Ecker JR. Exploiting the triple response of Arabidopsis to identify ethylene-related mutants. Plant Cell 1990; 2:513-523.

13 Rothenberg M, Ecker JR. Mutant analysis as an experimental approach towards understanding plant hormone action. Sem Dev Biol Plant Dev Genet 1993; 4:3-13.

14 Kieber JJ, Rothenberg M, Roman G, Feldmann KA, Ecker JR. CTR1, a negative regulator of the ethylene response pathway in Arabidopsis, encodes a member of the raf family of protein kinases. Cell 1993; 72:427-441.

15 Roman G, Ecker JR. Genetic analysis of a seedling stress response to ethylene in Arabidopsis. Philos Trans R Soc Lond B Biol Sci 1995; 350:75-81.

16 Zadnikova P, Petrasek J, Marhavy P, et al. Role of PIN-mediated auxin efflux in apical hook development of Arabidopsis thaliana. Development 2010; 137:607-617.

17 Vandenbussche F, Petrasek J, Zadnikova P, et al. The auxin influx carriers AUX1 and LAX3 are involved in auxin-ethylene interactions during apical hook development in Arabidopsis thaliana seedlings. Development 2010; 137:597-606.

18 Achard P, Vriezen WH, Van Der Straeten D, Harberd NP. Ethylene regulates Arabidopsis development via the modulation of DELLA protein growth repressor function. Plant Cell 2003; 15:2816-2825.

19 Sun TP, Gubler F. Molecular mechanism of gibberellin signaling in plants. Annu Rev Plant Biol 2004; 55:197-223.

20 Fu X, Richards DE, Ait-Ali T, et al. Gibberellin-mediated proteasome-dependent degradation of the barley DELLA protein SLN1 repressor. Plant Cell 2002; 14:3191-3200.

21 Silverstone AL, Jung HS, Dill A, Kawaide H, Kamiya Y, Sun TP. Repressing a repressor: gibberellin-induced rapid reduction of the RGA protein in Arabidopsis. Plant Cell 2001; 13:1555-1566.

22 Peng J, Richards DE, Hartley NM, et al. 'Green revolution' genes encode mutant gibberellin response modulators. Nature 1999; 400:256-261.

23 Dill A, Jung HS, Sun TP. The DELLA motif is essential for gibberellin-induced degradation of RGA. Proc Natl Acad Sci USA 2001; 98:14162-14167.

24 Zentella R, Zhang ZL, Park M, et al. Global analysis of della direct targets in early gibberellin signaling in Arabidopsis. Plant Cell 2007; 19:3037-3057.

25 Daviere JM, de Lucas M, Prat S. Transcriptional factor interaction: a central step in DELLA function. Curr Opin Genet Dev 2008; 18:295-303.

26 de Lucas M, Daviere JM, Rodriguez-Falcon M, et al. A molecular framework for light and gibberellin control of cell elongation. Nature 2008; 451:480-484.

27 Feng S, Martinez C, Gusmaroli G, et al. Coordinated regulation of Arabidopsis thaliana development by light and gibberellins. Nature 2008; 451:475-479.

28 Arnaud N, Girin T, Sorefan K, et al. Gibberellins control fruit patterning in Arabidopsis thaliana. Genes Dev 2010; 24:2127- 
2132.

29 Heo JO, Chang KS, Kim IA, et al. Funneling of gibberellin signaling by the GRAS transcription regulator scarecrowlike 3 in the Arabidopsis root. Proc Natl Acad Sci USA 2011; 108:2166-2171.

30 Zhang ZL, Ogawa M, Fleet CM, et al. Scarecrow-like 3 promotes gibberellin signaling by antagonizing master growth repressor DELLA in Arabidopsis. Proc Natl Acad Sci USA 2011; 108:2160-2165.

31 Hou X, Lee LY, Xia K, Yan Y, Yu H. DELLAs modulate jasmonate signaling via competitive binding to JAZs. Dev Cell 2010; 19:884-894.

32 Alonso JM, Stepanova AN, Solano R, et al. Five components of the ethylene-response pathway identified in a screen for weak ethylene-insensitive mutants in Arabidopsis. Proc Natl Acad Sci USA 2003; 100:2992-2997.

33 An F, Zhao Q, Ji Y, et al. Ethylene-induced stabilization of ETHYLENE INSENSITIVE3 and EIN3-LIKE1 is mediated by proteasomal degradation of EIN3 binding F-box 1 and 2 that requires EIN2 in Arabidopsis. Plant Cell 2010; 22:23842401.

34 Chao Q, Rothenberg M, Solano R, Roman G, Terzaghi W, Ecker JR. Activation of the ethylene gas response pathway in Arabidopsis by the nuclear protein ETHYLENE-INSENSITIVE3 and related proteins. Cell 1997; 89:1133-1144.

35 Zhao X, Yu X, Foo E, et al. A study of gibberellin homeostasis and cryptochrome-mediated blue light inhibition of hypocotyl elongation. Plant Physiol 2007; 145:106-118.

36 Schomburg FM, Bizzell CM, Lee DJ, Zeevaart JA, Amasino RM. Overexpression of a novel class of gibberellin 2-oxidases decreases gibberellin levels and creates dwarf plants. Plant Cell 2003; 15:151-163.

37 Tyler L, Thomas SG, Hu J, et al. Della proteins and gibberellin-regulated seed germination and floral development in Arabidopsis. Plant Physiol 2004; 135:1008-1019.

38 Ursin VM, Bradford KJ. Auxin and ethylene regulation of petiole epinasty in two developmental mutants of tomato, diageotropica and epinastic. Plant Physiol 1989; 90:1341-1346.

39 Cowling RJ, Harberd NP. Gibberellins control Arabidopsis hypocotyl growth via regulation of cellular elongation. J Exp Bot 1999; 50:1351-1357.

40 Jacobsen SE, Olszewski NE. Mutations at the SPINDLY locus of Arabidopsis alter gibberellin signal transduction. Plant Cell 1993; 5:887-896.

41 Montgomery J, Goldman S, Deikman J, Margossian L, Fischer RL. Identification of an ethylene-responsive region in the promoter of a fruit ripening gene. Proc Natl Acad Sci USA 1993; 90:5939-5943.

42 Stepanova AN, Hoyt JM, Hamilton AA, Alonso JM. A Link between ethylene and auxin uncovered by the characterization of two root-specific ethylene-insensitive mutants in Arabidopsis. Plant Cell 2005; 17:2230-2242.

43 Chen $\mathrm{H}$, Xue L, Chintamanani S, et al. ETHYLENE INSENSITIVE3 and ETHYLENE INSENSITIVE3-LIKE1 repress SALICYLIC ACID INDUCTION DEFICIENT2 expression to negatively regulate plant innate immunity in Arabidopsis. Plant Cell 2009; 21:2527-2540.

44 Solano R, Stepanova A, Chao Q, Ecker JR. Nuclear events in ethylene signaling: a transcriptional cascade mediated by ETHYLENE-INSENSITIVE3 and ETHYLENE-RESPONSEFACTOR1. Genes Dev 1998; 12:3703-3714.

45 Gallego-Bartolome J, Arana MV, Vandenbussche F, et al. Hierarchy of hormone action controlling apical hook development in Arabidopsis. Plant J 2011; 67:622-634.

46 Vriezen WH, Achard P, Harberd NP, Van Der Straeten D. Ethylene-mediated enhancement of apical hook formation in etiolated Arabidopsis thaliana seedlings is gibberellin dependent. Plant J 2004; 37:505-516.

47 De Grauwe L, Vandenbussche F, Tietz O, Palme K, Van Der Straeten D. Auxin, ethylene and brassinosteroids: tripartite control of growth in the Arabidopsis hypocotyl. Plant Cell Physiol 2005; 46:827-836.

48 Turner JG, Ellis C, Devoto A. The jasmonate signal pathway. Plant Cell 2002; 14 Suppl:S153-S164.

49 Leivar P, Tepperman JM, Monte E, Calderon RH, Liu TL, Quail PH. Definition of early transcriptional circuitry involved in light-induced reversal of PIF-imposed repression of photomorphogenesis in young Arabidopsis seedlings. Plant Cell 2009; 21:3535-3553.

50 Alonso JM, Hirayama T, Roman G, Nourizadeh S, Ecker JR. EIN2, a bifunctional transducer of ethylene and stress responses in Arabidopsis. Science 1999; 284:2148-2152.

51 Cheng H, Qin L, Lee S, et al. Gibberellin regulates Arabidopsis floral development via suppression of DELLA protein function. Development 2004; 131:1055-1064.

52 Lee S, Cheng H, King KE, et al. Gibberellin regulates Arabidopsis seed germination via RGL2, a GAI/RGA-like gene whose expression is up-regulated following imbibition. Genes Dev 2002; 16:646-658.

53 Bechtold N, Pelletier G. In planta Agrobacterium-mediated transformation of adult Arabidopsis thaliana plants by vacuum infiltration. Methods Mol Biol 1998; 82:259-266.

(Supplementary information is linked to the online version of the paper on the Cell Research website.) 